IAU Colloguium 164: Radio Emission from Galactic and Extragalactic Compact Sources

ASP Conference Series, Vol. 144, 1998

J. A. Zensus, G. B. Taylor, \& J. M. Wrobel (eds.)

\title{
Unification of Radio Galaxies
}

\author{
M. H. Cohen \& P. M. Ogle \\ Caltech, Pasadena, CA 91125, U.S.A.
}

H. D. Tran

IGPP, Lawrence Livermore National Laboratory, Livermore, CA 94550, U.S.A.

R. W. Goodrich

W.M. Keck Observatory, Kamuela, HI 96743, U.S.A.

\begin{abstract}
Many FR 2 narrow-line radio galaxies also display polarized broad lines. The broad-line region is hidden from direct view and is seen by reflection (ie scattering). In these objects the spectral classification is controlled by the aspect at which they are viewed.
\end{abstract}

Radio-loud quasars and FR 2 radio galaxies (RG) are highly anisotropic, and it has been hypothesized that they form a single population sorted by aspect: the quasars are seen nearly end-on and the RG are seen at higher inclinations. The RG are further subdivided according to their optical spectra; type 1 (BLRG) are dominated by broad permitted lines and type 2 (NLRG) are dominated by strong narrow forbidden lines. Following the picture commonly accepted for Seyfert galaxies, all (or most) of these RG should have a broad-line region (BLR) inside an opaque "torus", and the apparent spectral classification will depend on aspect. In the equatorial plane the BLR is hidden from view and the spectrum is that of an NLRG. At intermediate angles we see a BLRG, and when seen nearly end-on it is a quasar (eg Barthel 1989). Radiation from the BLR will escape along the axis and can be scattered into our line-of-sight by electrons or dust. This scattered light is polarized and so the spectrum of a FR 2 NLRG should show polarized broad lines. A few examples of this have been published, 3C 234 (Antonucci 1984, Tran, Cohen, \& Goodrich 1995), 3C 321 (Young et al. 1996), and Cygnus A (Ogle et al. 1997).

We have observed a number of FR 2 RG with the Keck telescope, using LRIS (Oke et al. 1995) and the polarimetry optics. The objective has been to test the unification picture described above, and to study the nuclear regions of the galaxies. Figure 1 shows spectra for 3 galaxies. The left panel is the total flux and the right panel is the polarized flux, calculated by multiplying the measured linear polarization $\left(\sqrt{q^{2}+u^{2}}\right)$ by the total flux. 3C 445 is a wellknown BLRG (Antonucci 1984) but the polarized flux contains an additional, much wider component, which can be seen in Fig. 1. (These spectra of 3C 445 were obtained at Palomar Observatory.) 3C 234 shows a weak broad line in total flux whereas the polarized flux is dominated by broad $\mathrm{H} \alpha, \mathrm{H} \beta$ and $\mathrm{H} \gamma .3 \mathrm{C} 33$ is a classic NLRG and its polarized spectrum is quasar-like, with very strong and broad hydrogen lines. In all objects the narrow oxygen lines are less polarized than the broad hydrogen lines and the continuum.

We have studied a total of 12 FR 2 RG, of which 8 are NLRG. One NLRG shows no polarization to a limit of $0.3 \%$; another, weakly polarized, does not show broad $\mathrm{H} \alpha$. The others do show broad $\mathrm{H} \alpha$ in polarized light. We conclude that many NLRG contain a hidden broad-line region. Polarimetric imaging studies (eg Draper et al. 1993; Ogle et al. 1997) show that the polarization often arises in an extended conical reflection nebula which is illuminated by a central point source. All this supports the unification hypothesis, that the nucleus con- 


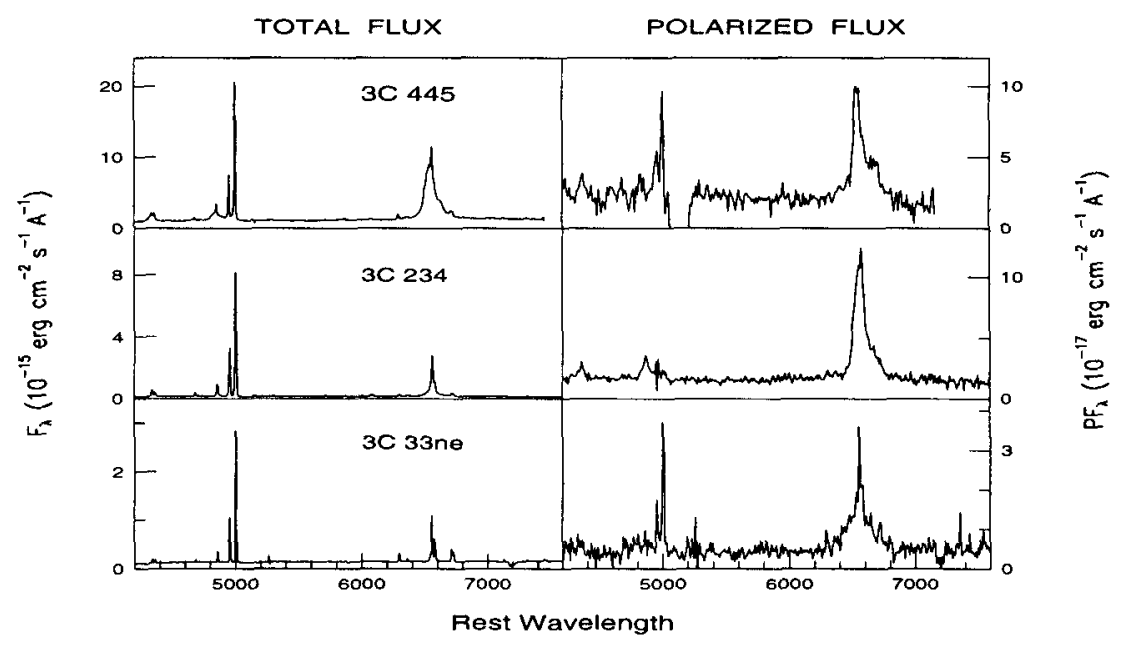

Figure 1. Total and polarized flux for three FR 2 radio galaxies. 3C 445 was observed at Palomar Observatory and the gap is due to the dichroic reflector.

tains an AGN hidden by an opaque torus so that the observed spectrum depends on aspect. NLRG, BLRG, and quasars are seen at progressively decreasing inclination of the pole. In some cases the inferred luminosity is sufficient that the $\mathrm{RG}$ would be called a quasar if the central region were seen directly.

Acknowledgments. The W.M. Keck Observatory is operated as a scientific partnership between the California Institute of Technology and the University of California; it was made possible by the generous support of the W.M. Keck foundation. This research has made use of the NASA/IPAC Extragalactic Database (NED).

\section{References}

Antonucci, S. 1984. ApJ, 278, 499-520.

Barthel, P. D. 1989. ApJ, 336, 606-611.

Draper, P. W., Scarrott, S.M., \& Tadhunter, C.N. 1993. MNRAS, 262, 1029-1036.

Ogle, P. M., et al. 1997. ApJ, 482, L37-40.

Oke, J. B., et al. 1995. PASP, 107, 375-385.

Tran, H. D., Cohen, M. H., \& Goodrich, R. W. 1995. AJ, 110, 2597-2604.

Young, S., et al. 1996. MNRAS, 279, L72-76. 